University of Nebraska - Lincoln

DigitalCommons@University of Nebraska - Lincoln

October 1987

\title{
Optimization of effective nonlinear coefficients for second- harmonic generation in molecular crystals
}

\author{
K.M. White \\ University of Nebraska - Lincoln \\ Craig J. Eckhardt \\ University of Nebraska - Lincoln, ceckhardt1@unl.edu
}

Follow this and additional works at: https://digitalcommons.unl.edu/chemistryeckhardt

Part of the Chemistry Commons

White, K.M. and Eckhardt, Craig J., "Optimization of effective nonlinear coefficients for second-harmonic generation in molecular crystals" (1987). Craig J. Eckhardt Publications. 26.

https://digitalcommons.unl.edu/chemistryeckhardt/26

This Article is brought to you for free and open access by the Published Research - Department of Chemistry at DigitalCommons@University of Nebraska - Lincoln. It has been accepted for inclusion in Craig J. Eckhardt Publications by an authorized administrator of DigitalCommons@University of Nebraska - Lincoln. 


\title{
Optimization of effective nonlinear coefficients for second-harmonic generation in molecular crystals
}

\author{
K. M. White and C. J. Eckhardt \\ Department of Chemistry, University of Nebraska, Lincoln, Nebraska 68588-0304
}

(Received 9 March 1987; revised manuscript received 1 June 1987)

\begin{abstract}
Conditions for the design of organic solids capable of optimum nonlinear optical behavior are investigated. Generalized expressions describing optimum phase-matching conditions in crystals of monoclinic symmetry and having one-dimensional hyperpolarizabilities are obtained. The results are applied to specific organic molecular solids. An anisotropic in-plane molecular polarizability is shown to provide the best condition for second-harmonic generation in these systems.
\end{abstract}

\section{INTRODUCTION}

In recent years, the search for highly efficient materials capable of supporting second-harmonic generation (SHG) has turned to organic compounds with considerable success. ${ }^{1-10}$ Using $p$-nitroaniline as a prototype, several molecules have been synthesized whose crystals exhibit SHG intensities that exceed those seen in urea as well as in many inorganic crystals having nonlinear optical responses. In synthesizing these molecules, researchers have sought to implement those physical properties that increase the second-order susceptibility of the crystal which gives rise to the SHG effect.

Several factors are involved in determining the second-order susceptibility in organic crystals. Efforts to enhance a major factor, the molecular hyperpolarizability, have concentrated on creating a large, intramolecular charge-transfer dipole, thereby making the hyperpolarizability nearly one dimensional. In an extensive theoretical study, ${ }^{11}$ Zyss and Oudar took advantage of the oriented gas approximation, which can often be implemented in organic solids, to derive the expressions that relate the hyperpolarizabilities of the molecules in the crystal to the second-order susceptibility tensor whose elements are represented by the $d_{I J K}$ coefficients. With this information in hand, they then determined, for all allowed nonlinear crystal symmetries, the molecular orientations that would optimize the $d_{I J K}$ coefficients of materials having one-dimensional hyperpolarizabilities. This theoretical work represented a significant step forward in the field of nonlinear optics since it provided guidelines which can be utilized in developing and preparing compounds that are capable of more efficient SHG as well as other nonlinear effects. Indeed, since the report of the study, two organic molecules have been discovered which apparently provide excellent examples of the idealized model. The crystal structures of $N$-(4nitrophenyl)-( $L$ )-prolinol (NPP) $)^{12,13}$ and 2 - $(N$-prolinol $)$ 5-nitropyridine (PNP), ${ }^{14,15}$ both of which produce large SHG intensities, have been found to exhibit molecular packing that is very similar to the proposed optimal orientations.

While large $d_{I J K}$ coefficients in an organic solid are important in determining the efficiency of the SHG it produces, their effective values may be significantly reduced when collinear phase matching is employed to increase the intensity of the frequency-doubled output. ${ }^{16}$ Furthermore, if noncritical phase-matching (NCPM) conditions cannot be employed, the SHG efficiency will not be at a maximum. In the search for new organic nonlinear optical materials, the phase-matching properties of the crystals have been considered a posteriori, and less effort has been directed toward developing materials in which the effective $d_{I J K}$ coefficients are optimized by means of nearly ideal phase-matching conditions. No doubt this lack of emphasis is due to the presently insufficient knowledge of how to precisely control the structure of a van der Waals crystal by means of synthetic modifications of the individual molecules that comprise it.

Some of the principles behind the problem of phasematched SHG have been addressed previously. ${ }^{16,17}$ In the ideal case, the optimal collinear phase-matched propagation direction should coincide with a principal dielectric axis of the crystal to give NCPM. The requirements for this condition were derived by Zyss and Oudar ${ }^{11}$ in terms of molecular polarizabilities for a biaxial crystal composed of molecules having one-dimensional hyperpolarizabilities and belonging to the point group 2. Examination of the recently corrected result ${ }^{18}$ not only shows that optimized phase-matching conditions are theoretically accessible to the model molecule used in the derivation, but also suggests application to an even wider range of organic molecular crystals demonstrating large SHG responses.

The intent of this paper is to extend the framework established by Zyss and Oudar for the design of organic solids to produce highly efficient SHG. This is accomplished by providing a more general expression for optimal phase-matched SHG which is applicable to several systems. The effects of crystal optics on the electric fields involved in this nonlinear phenomenon will also be considered. The results will be interpreted for the purpose of clarifying the principles that may be followed to design organic crystals for SHG in which collinear phase matching takes full advantage of large $d_{I J K}$ coefficients. Finally, the results will be applied to the NPP and PNP crystals and their derivatives to provide examples of pos- 
sible model systems that could be used to test the proposed theories.

\section{OPTIMUM COLLINEAR PHASE MATCHING}

In order to consider optimized collinear phase matching in crystal systems other than the one dealt with previously, ${ }^{11,18}$ the derived conditions will be adapted for a more general structure belonging to the crystal point group 2. This particular point group is a logical choice since it has been determined that some $80 \%$ of the organic crystals in which SHG is allowed by symmetry belong to either the $P 2_{1}$ or $P 2{ }_{1} 2_{1} 2_{1}$ space groups. ${ }^{15}$ Moreover, the optimized phase-matchable coefficient obtained for a system having a given one-dimensional molecular hyperpolarizability is largest for crystals in this point group. ${ }^{11}$ An added advantage of performing the derivation for point group 2 is that it is equally well applied to crystals in point group $m$.

Using the crystal axial system in Fig. 1 in which the $Y Z$ plane contains the one-dimensional molecular hyperpolarizability $\beta_{u u u}$ oriented at an angle $\theta$ to the symmetry-fixed $Y$ axis, it has been shown ${ }^{11}$ that the optimal phase-matchable nonlinear coefficient in point group 2 is $d_{Y Z Z}$. It is given by

$$
d_{Y Z Z}=N \beta_{u u u} \cos \theta \sin ^{2} \theta,
$$

where $N$ is a factor that accounts for local fields and the number of molecules per unit volume in the crystal. The value of $d_{Y Z Z}$ is at a maximum when $\theta=54.74^{\circ}$, while its effective value for collinear phase-matched SHG is largest when the fundamental and harmonic waves are polarized along the $Z$ and $Y$ axes, respectively, and the $X$ axis is the phase-matched direction. If the $X$ axis is not also a principal dielectric axis of the crystal, however, the SHG efficiency is not fully maximized.

The desire to have a principal dielectric axis for a phase-matching propagation direction can be understood by considering the effects of the optical indicatrix in anisotropic crystals on the electric fields involved in col-

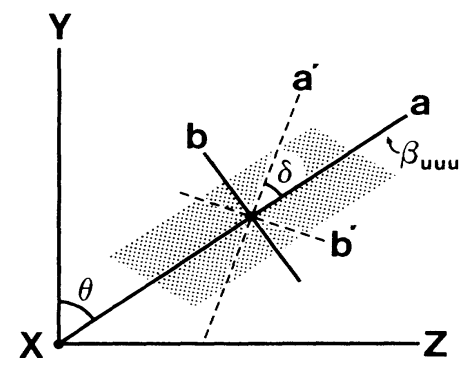

FIG. 1. Axial system used to determine the molecular polarizability relation that optimizes noncritical phase matching in a crystal of point group 2 where the average molecular planes are normal to the ideal phase-matched propagation direction $X$. The $c$ axis is normal to the molecular plane and the $a^{\prime}$ and $b^{\prime}$ axes are the principal in-plane polarizability axes for the molecule. The one-dimensional molecular hyperpolarizability $\beta_{u u u}$ is oriented along the molecular $a$ axis. linear phase matching. As an example, we consider phase matching involving the nonlinear coefficient $d_{Y Z Z}$ in the system described above in which the $X$ axis is the phase-matched propagation direction but is not a principal dielectric axis. Figure 2 shows the intersections of two hypothetical refractive index surfaces of this biaxial crystal with the $X^{\prime} Z^{\prime}$ plane, where $X^{\prime}$ and $Z^{\prime}$ are principal dielectric axes. The dashed curve corresponds to the fundamental frequency and the solid curve to the harmonic frequency. The phase-matching direction, determined by the intersection of the two curves, is indicated by the wave vector $W$ along the $X$ axis. Since the harmonic wave is polarized along the $Y$ axis, its Poynting vector coincides with the wave vector. However, the electric field of the fundamental wave is polarized in the $X^{\prime} Z^{\prime}$ plane along the direction tangent to its wave-vector curve where it intersects $W$. Consequently, the Poynting vector $F$ of the fundamental wave is separated from the wave vector by an angle $\psi$.

The crystal optics reduce the efficiency of the SHG in two ways. First, since the electric field of the fundamental wave inside the crystal makes an angle $\psi$ with the $Z$ axis, the effective value of $d_{Y Z Z}$ is given by

$$
d_{\mathrm{eff}}=d_{Y Z Z} \cos ^{2} \psi
$$

The $d_{\text {eff }}$ value thus derived may differ significantly from that obtained when refraction of the fundamental wave is neglected. This bending also gives rise to the beam walkoff angle $\psi$. As a result, the fundamental and harmonic waves do not remain coincident and, in general, this condition will also lower the efficiency of the phasematched SHG produced in the crystal. On the other hand, if the phase-matched direction were to lie along a principal dielectric axis of the crystal, neither of the effects described above would occur and $d_{\text {eff }}$ would equal $d_{Y Z Z}$.

A number of organic crystals with large SHG responses $^{12-15,19,20}$ have their constituent molecules oriented with mean molecular planes parallel to the $Y Z$

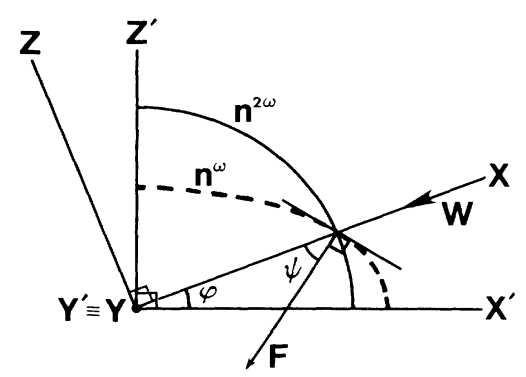

FIG. 2. The effect of crystal optics on type-I phase matching involving the $d_{Y Z Z}$ nonlinear coefficient for a hypothetical crystal in the point group 2. The solid and dashed curves show the intersection of the index surfaces of the harmonic and fundamental waves, respectively, with the crystal $X^{\prime} Z^{\prime}$ principal plane. The phase-matched propagation direction is indicated by the wave vector $W$. For this case, the electric vector of the fundamental wave is polarized perpendicular to the Poynting vector $F$ and is not normal to its wave vector. 
plane shown in Fig. 1. We define the molecular axial system so that the $c$ axis is normal to the average molecular plane. The $a$ axis, which coincides with the orientation of $\beta_{u u u}$, lies in this plane and the $b$ axis is orthogonal to the other two axes. If the molecule is assumed to be pseudoplanar, then the $c$ axis will also be a principal polarizability axis of the molecule. This condition alone necessitates that the $X$ axis be a principal dielectric axis of the crystal. ${ }^{21}$ The requirement of the previous derivation $^{11,18}$ that the in-plane polarizability of the molecule be isotropic is thus removed. One of the crystals having mean molecular planes in a similar orientation is 2methyl-4-nitroaniline (MNA), ${ }^{19,20}$ in which the dielectric axis has, in fact, been found to be only $8^{\circ}$ off of the normal to the planes.

We are now in a position to derive the molecular polarizability relation that will also make the $X$ axis a phase-matching direction in the crystal. In terms of the crystal refractive indices, the phase-matching requirement is

$$
n_{Y}^{2 \omega}=n_{Z}^{\omega}
$$

Defining $a^{\prime}$ and $b^{\prime}$ as the principal in-plane polarizability axes for the molecule and using the formalism of Ref. 11 , Eq. (3) leads to

$$
\begin{aligned}
\alpha_{a^{\prime} a^{\prime}}^{2 \omega} \cos ^{2}(\theta-\delta) & +\alpha_{b^{\prime} b^{\prime}}^{2 \omega} \sin ^{2}(\theta-\delta) \\
= & \alpha_{a^{\prime} a^{\prime}}^{\omega} \sin ^{2}(\theta-\delta)+\alpha_{b^{\prime} b^{\prime}}^{\omega} \cos ^{2}(\theta-\delta)
\end{aligned}
$$

which, in turn, reduces to

$$
\tan ^{2}(\theta-\delta)=\left(\alpha_{a^{\prime} a^{\prime}}^{2 \omega}-\alpha_{b^{\prime} b^{\prime}}^{\omega}\right) /\left(\alpha_{a^{\prime} a^{\prime}}^{\omega}-\alpha_{b^{\prime} b^{\prime}}^{2 \omega}\right)
$$

Since the left-hand side of Eq. (5) is positive, it is seen that not only is an isotropic in-plane molecular polarizability not necessary, but should actually be avoided. Otherwise, given that $\alpha_{a^{\prime} a^{\prime}}^{\omega}=\alpha_{b^{\prime} b^{\prime}}^{\omega}, \quad \alpha_{a^{\prime} a^{\prime}}^{2 \omega}=\alpha_{b^{\prime} b^{\prime}}^{2 \omega}, \quad$ and $\alpha_{a^{\prime} a^{\prime}}^{2 \omega}>\alpha_{a^{\prime} a^{\prime}}^{\omega}$, the right-hand side of the equation would necessarily be negative.

By setting $\delta$ equal to zero, Eq. (5) can also be used for crystals in which the mean molecular planes are perpendicular to the crystal $Y Z$ plane, provided that there exists a principal molecular polarizability axis that is also perpendicular to $Y Z$. For example, molecules having a twofold symmetry axis in the same direction as $\beta_{u u u}$ or an isotropic in-plane molecular polarizability would fulfill this criterion. In the latter case, the relation is equivalent to the result obtained in Ref. 18.

\section{TEST MODELS: NPP AND PNP}

In order to apply the result derived in Eq. (5), a model nonlinear optical material must be found that will allow the design of the in-plane linear polarizability of the constituent molecules with the retention of the crystal structure. In such a system, the orientation and magnitude of the principal polarizability axes in the molecules could be adjusted in order to make the $X$ axis (Fig. 1) a phasematchable direction of the crystal. This would enable the SHG efficiency of the material to be increased without lowering the effective value of the $d_{Y Z Z}$ coefficient.
The crystal structures of NPP (Refs. 12 and 13) and PNP (Refs. 14 and 15) suggest an example of a possible model system. The structures show that, with respect to SHG, both of these molecules are optimally oriented for a system belonging to point group 2 and composed of molecules having one-dimensional hyperpolarizabilities. Consequently, it is expected that their $d_{Y Z Z}$ coefficients make maximum use of the molecular hyperpolarizabilities and are responsible for the large SHG responses exhibited by these organic solids. In addition, the two crystal structures are isomorphous. The only difference between NPP and PNP is the exchange of a nitrogen for a carbon atom in the benzene ring of the molecules. As a result, it is possible to test the above derivation of ideal phase-matching conditions by determining the phasematching directions in these crystals and observing how they may be affected by the anistropic polarizabilities in each of the molecules. Then, by appropriate substitution of the atoms comprising the benzene ring, a derivative may be found that will satisfy Eq. (5) to give a highly efficient material for SHG.

Not all problems have been overcome in using the NPP and PNP crystals for a model system, however. It has been suggested that the one-dimensional hyperpolarizability approximation used herein may begin to fail in PNP since the introduction of the nitrogen atom puts the ground-state dipole some $20^{\circ}$ off of the long axis of the molecule. ${ }^{14}$ The possibility of synthesizing the pyrimidine derivative that would make the molecular long axis a pseudobinary axis as in NPP may remedy the situation and still satisfy Eq. (5). Indeed, all possible derivatives should be examined not only to find a crystal that optimizes the aspects of collinear phase matching that are discussed here, but also to completely test the relationships derived.

It is important to realize that only type-I phase matching has been dealt with in the derivations as well as in the examples. Recent experimental evidence ${ }^{22,23}$ indicates that the $X$ axis is a type-II phase-matched direction in NPP. This does not alter the above results, since NPP has only been used as an example of a possible model system. In fact, an expression analogous to Eq. (5) can be derived for type-II phase matching in the crystal system used here. Based on the type-II phasematching condition

$$
n_{Z}^{2 \omega}=\left(n_{Z}^{\omega}+n_{Y}^{\omega}\right) / 2
$$

one obtains

$$
\tan ^{2}(\theta-\delta)=\frac{\left[\alpha_{b^{\prime} b^{\prime}}^{2 \omega}-\left(\alpha_{a^{\prime} a^{\prime}}^{\omega}+\alpha_{b^{\prime} b^{\prime}}^{\omega}\right) / 2\right]}{\left[\left(\alpha_{a^{\prime} a^{\prime}}^{\omega}+\alpha_{b^{\prime} b^{\prime}}^{\omega}\right) / 2-\alpha_{a^{\prime} a^{\prime}}^{2 \omega}\right]}
$$

This expression is not as easily applied as in the type-I phase-matching case, however. There, the right-hand side of Eq. (5) can be increased (decreased) to equal the left-hand side by simply increasing (decreasing) the polarizability component along the molecular $b^{\prime}$ axis. On the other hand, the additional terms in both the numerator and denominator of Eq. (7) make an adjustment more complex in the case of type-II phase matching.

Although the refractive indices have not been mea- 
sured in the NPP and PNP crystals, their molecular orientations suggest that $n_{X}$ is less than both $n_{Y}$ and $n_{Z}$. Therefore, assuming that $d_{Y Z Z}$ is the only phasematchable nonlinear coefficient in these crystals, only type-I noncritical phase matching is possible when $n_{Z}>n_{Y}$, while only type-II noncritical phase matching is possible for $n_{Y}>n_{Z} \cdot{ }^{17}$ Should $n_{Y}=n_{Z}$, which would occur for $\alpha_{a^{\prime} a^{\prime}}=\alpha_{b^{\prime} b^{\prime}}=\alpha_{\|}$, then noncritical phase matching would not be possible.

\section{CONCLUSION}

A generalized molecular polarizability expression that will lead to optimum noncritical phase-matching conditions in crystals belonging to the point group 2 and having one-dimensional hyperpolarizabilities has been obtained. The relation has been extended to apply to crystals in which the mean molecular planes are normal to the ideal phase-matched propagation direction. It is shown that an anisotropic in-plane molecular polarizability is most advantageous for SHG in these systems. Possible fulfillment of the requirements is considered for the series of crystals of NPP, PNP, and their derivatives. The isomorphic crystals of NPP and PNP suggest that in-plane polarizabilities may be adjusted in some SHG active molecules without altering the crystal structure.

The more general expression is also applicable to crystals in the point group $m$ and can be adapted for use in type-II phase matching. An appropriate definition of molecular axes will reduce it to the result derived for the case treated previously. ${ }^{11,18}$

Employing the approach of Zyss and Oudar has permitted a refinement of the conditions required of molecules to form crystals suitable for efficient SHG. This has made it possible to specify the kinds of molecules which should provide the best candidates for SHG. Unfortunately, the assumption must be made that the molecules will pack in one of the assumed space groups. The paucity of understanding of the principles for the synthesis of crystals - organic crystals in particular - is still a major obstacle to the development of such materials. A major goal must be the development of a synthetic chemistry for solids which is as powerful as that which is available for the creation of molecules.

\section{ACKNOWLEDGMENT}

The authors would like to acknowledge financial support from 3M Company.
${ }^{1}$ Nonlinear Optical Properties of Organic and Polymeric Materials, edited by D. J. Williams, ACS Symposium Series No. 233 (American Chemical Society, Washington, D.C. 1983).

${ }^{2}$ D. J. Williams, Angew. Chem. Int. Ed. Engl. 23, 690 (1984).

${ }^{3}$ G. R. Meredith, J. G. VanDusen, and D. J. Williams, Macromolecules 15, 1385 (1982).

${ }^{4}$ G. R. Meredith, D. J. Williams, S. N. Fishman, E. S. Goldbrecht, and V. A. Krongauz, J. Phys. Chem. 87, 1697 (1983).

${ }^{5}$ J. Zyss, J. Non-Cryst. Solids 47, 211 (1982).

6J. L. Oudar and R. Hierle, J. Appl. Phys. 48, 2699 (1977).

${ }^{7}$ B. F. Levine, C. G. Bethea, C. D. Thurmond, R. T. Lynch, and J. L. Bernstein, J. Appl. Phys. 50, 2523 (1979).

${ }^{8}$ J. Zyss, D. S. Chemla, and J. F. Nicoud, J. Chem. Phys. 74, 4800 (1981).

${ }^{9}$ M. Sigelle, J. Zyss, and R. Hierle, J. Non-Cryst. Solids 47, 287 (1982).

${ }^{10}$ C. Chen and G. Liu, Annu. Rev. Mater. Sci. 16, 203 (1986).

${ }^{11}$ J. Zyss and J. L. Oudar, Phys. Rev. A 26, 2028 (1982).

${ }^{12}$ J. Zyss, J. F. Nicoud, and M. Coquillay, J. Chem. Phys. 81,
4160 (1984).

${ }^{13}$ J. Badan, R. Hierle, A. Perigaud, and J. Zyss, in Ref. 1, pp. $81-107$.

${ }^{14}$ R. J. Twieg and C. W. Dirk, J. Chem. Phys. 85, 3537 (1986).

${ }^{15}$ R. J. Twieg and K. Jain, in Ref. 1, pp. 57-80.

${ }^{16} \mathrm{~F}$. Zernike and J. E. Midwinter, Applied Nonlinear Optics (Wiley, New York, 1973).

${ }^{17}$ M. V. Hobden, J. Appl. Phys. 38, 4365 (1967).

${ }^{18}$ K. M. White and C. J. Eckhardt, Phys. Rev. A (to be published).

${ }^{19}$ G. F. Lipscomb, A. F. Garito, and R. S. Narang, J. Chem. Phys. 75, 1509 (1981).

${ }^{20}$ A. F. Garito, K. D. Singer, and C. C. Teng, in Ref. 1, pp. $1-26$.

${ }^{21}$ J. L. Oudar and J. Zyss, Phys. Rev. A 26, 2016 (1982).

22J. Zyss and G. Tsoucaris, Mol. Cryst. Liq. Cryst. 137, 303 (1986).

${ }^{23}$ I. Ledoux, D. Josse, P. Vidakovic, and J. Zyss, Opt. Eng. 25, 202 (1986). 